\title{
Safety and Belonging in the Field: A Checklist for Educators
}

Sarah E. Greene ${ }^{1 a^{*}}$, Gwen S. Antell ${ }^{2}$, Jake Atterby ${ }^{1 \mathrm{b \dagger}}$, Rehemat Bhatia ${ }^{3 a \dagger}$, Emma M. Dunne $^{1 \text { at }}$, Sam Giles ${ }^{1 a \dagger}$, Sebastian S. Groh ${ }^{4 c t}$, Emma M. Hanson ${ }^{1 a \dagger}$, Jason Hilton ${ }^{1 \mathrm{~d} \dagger}$, Hazel Knight $^{1 \mathrm{at}}$, Peter Kraftl ${ }^{1 \mathrm{d \dagger}}$, Esme Morgan ${ }^{1 \mathrm{e} \dagger}$, Isobel Rhodes ${ }^{1 \mathrm{a} \dagger}$, Francisca G.T. Rockey ${ }^{5 \mathrm{a} \dagger}$, Shivani Singh ${ }^{1 \mathrm{at}}$, Carl T. Stevenson ${ }^{1 \mathrm{~d} \dagger}$, Simiao Sun ${ }^{1 \mathrm{at}}$, Bridget A. Warren ${ }^{1 \dagger}$, James R. Wheeley $^{1 \mathrm{~d} \dagger}$, Kweku A. Yamoah ${ }^{1 \mathrm{~d} \dagger}$

${ }^{1}$ School of Geography, Earth and Environmental Sciences, University of Birmingham

${ }^{2}$ Department of Earth Sciences, University of Oxford

${ }^{3}$ Unaffiliated

${ }^{4}$ Department of Earth Sciences, University College London

${ }^{5}$ Black Geographers

${ }^{a}$ she/her; ${ }^{b}$ he/they; ${ }^{c}$ they/he; ${ }^{d}$ he/him; ${ }^{\text {eshe/they/he }}$

"Corresponding author. Email: s.e.greene@bham.ac.uk; Twitter: @carbonatefan

†These authors are listed alphabetically.

\section{Abstract}

Ensuring taught fieldwork is a positive, generative, collective, and valuable experience for all participants requires considerations beyond course content. To guarantee safety and belonging, participants' identities (backgrounds and protected characteristics) must be considered as a part of fieldwork planning and implementation. Furthermore, getting fieldwork right is an important step in disrupting the ongoing cycle of exclusion of participants from marginalised demographics. This document aims to provide those involved in field teaching in Geography, Earth, and Environmental Sciences (GEES) disciplines with a brief overview about how identity affects experiences of taught fieldwork, as well as some general tips and a practical checklist for creating a safe learning environment for all staff, demonstrators, and students in the field. 
This paper is a non-peer reviewed preprint submitted to EarthArXiv.

\section{Table of Contents}

Abstract

1. Author Positionality Statement

2. Motivation

2.1 Safety

2.2 Belonging

3. General Tips

3.1 Acknowledge Participants' Identities and Backgrounds

3.2 Invite Participants' Questions

3.3 Distribute Detailed Information Early

4. Checklist

4.1 Advance Preparation

4.1.1 Training

4.1.2 Code of Conduct

4.1.3 Health, Safety, Hazards and Risks

4.1.4 Resources

4.2 Fieldwork Planning

4.2.1 Choosing a Destination

4.2.2 Permission/Ethics

4.2.3 Accessibility

4.2.4 Housing Accommodation

4.2.5 Scheduling

4.2.6 Health, Safety, Hazards, and Risks

4.2.7 Code of Conduct Amendments

4.2.8 Combating Isolation

4.2.9 Staffing

4.2.10 Learning Objectives

4.2.11 Logistics, Itinerary, and Kit

4.2.12 Communication and Delegation

4.2.13 Create Inclusive Field Guides

4.3 Pre-Fieldwork Student Briefings

4.3.1 Offer Advance Small Group or Private Meetings

4.3.2 Set an Inclusive and Collaborative Tone

4.3.3 Review Health, Safety, Hazards, and Risks

4.3.4 Review Logistics, Itinerary, and Kit

4.3.5 Follow Up

$\underline{4.4 \ln \text { the Field }}$

4.4.1 General Conduct

4.4.2 Accessible Course Content Delivery

4.4.3 Socialising and Alcohol

4.4.4 Marking

4.4.5 Contingency Expenses 
This paper is a non-peer reviewed preprint submitted to EarthArXiv.

\subsection{On Return}

4.5.1 Respect Participants' Privacy

4.5.2 Reflect and Revise

4.5.3 Iterate and Self-Educate

$\underline{5 . \text { Acknowledgements }}$

6. Further Resources

\section{References}

8. Printable Checklist: Safety and Belonging in the Field 


\section{Author Positionality Statement}

The authors comprise current and former staff and students in UK Higher Education spanning a variety of GEES disciplines. The checklist began as a University of Birmingham (UoB) Earth Sciences staff-student brainstorm; additional internal and external authors were recruited whose identities and backgrounds were underrepresented in the initial author list. Some of us have only experienced taught fieldwork from an undergraduate perspective, some have experience as postgraduate demonstrators, and others have been staffing or leading field courses for years to decades. Our best practice recommendations are informed by our own personal experiences of fieldwork, both in the UK and abroad, and by further undergraduate student testimonials from the School of Geography, Earth and Environmental Science at UoB (Kraftl et al., 2021; Singh et al., in prep.).

While we cannot speak to the full spectrum of possible individualised experiences, we have taken care to abide by a "Nothing About Us Without Us" ethos. Individual authors describe themselves as or self-identify with characteristics including: genderfluid, genderless, man, woman; cisgender, transgender; dyadic, intersex; aromantic, asexual, biromantic, bisexual, gay, polyamorous, queer, straight; Asian, Black, South Asian, white; agnostic, Christian, Jewish, Muslim, nonreligious; autistic, chronically ill, disabled, dyslexic, neurodiverse, neurotypical, nondisabled, restricted mobility; British, international; middle class background, working class background, first-generation university student, second-generation academic; no caring responsibilities, parent; fat, fit, not 'outdoorsy', thin, unfit.

At time of writing, SARS-CoV-2 presents significant practical challenges and safety concerns for taught fieldwork. Identity (e.g., age, race, class, disability status, caring responsibilities) can modulate these challenges and concerns, with individuals from many historically excluded demographics bearing disproportionate risks and burdens. A comprehensive enumeration of risks and risk management strategies related to the global pandemic falls outside the scope of this document. The authors draw on field experiences primarily from before 2020 and with the intention that checklist items will remain applicable long-term, in future years when public health threats may be less severe. Nevertheless, principles discussed herein (e.g., risk assessment guidelines, attention to local regulations, financial support, and accommodations for people with chronic health conditions like immunocompromised status or long COVID) can help guide fieldwork planning around SARS-CoV-2.

\section{Motivation}

\subsection{Safety}

Field teaching is highly valued in GEES disciplines (Mogk and Goodwin, 2012; Petcovic et al., 2014) and has demonstrable positive outcomes for learning (e.g., Elkins and Elkins, 2018), but also presents a number of barriers to safe participation and engagement, particularly for individuals whose identities have been historically excluded from the discipline (Giles et al., 2020; Pickrell, 2020). Ensuring the physical safety of every participant requires special consideration of a number of identities, backgrounds, and protected characteristics (Demery and Pipkin, 2020), including gender identity/expression and 
sexuality (Clancy et al., 2014; Black, 2019; Olcott and Downen, 2020; Jackson, 2021), race and ethnicity (Hughes, 2016; Anadu et al., 2020; Chaudhary and Berhe, 2020; Viglione, 2020; Ali et al., 2021), disability, neurodiversity, and mental ill health (Gilley et al., 2015; John and Khan, 2018; Tucker and Horton, 2018; Batty, 2020) and the intersection of these characteristics (Scarlett, 2021). The safety of each and every participant is the responsibility of fieldwork staff and particularly the field leader, who have "unparalleled power to mitigate harm in environments they oversee" (Cooperdock et al., 2021, p.1).

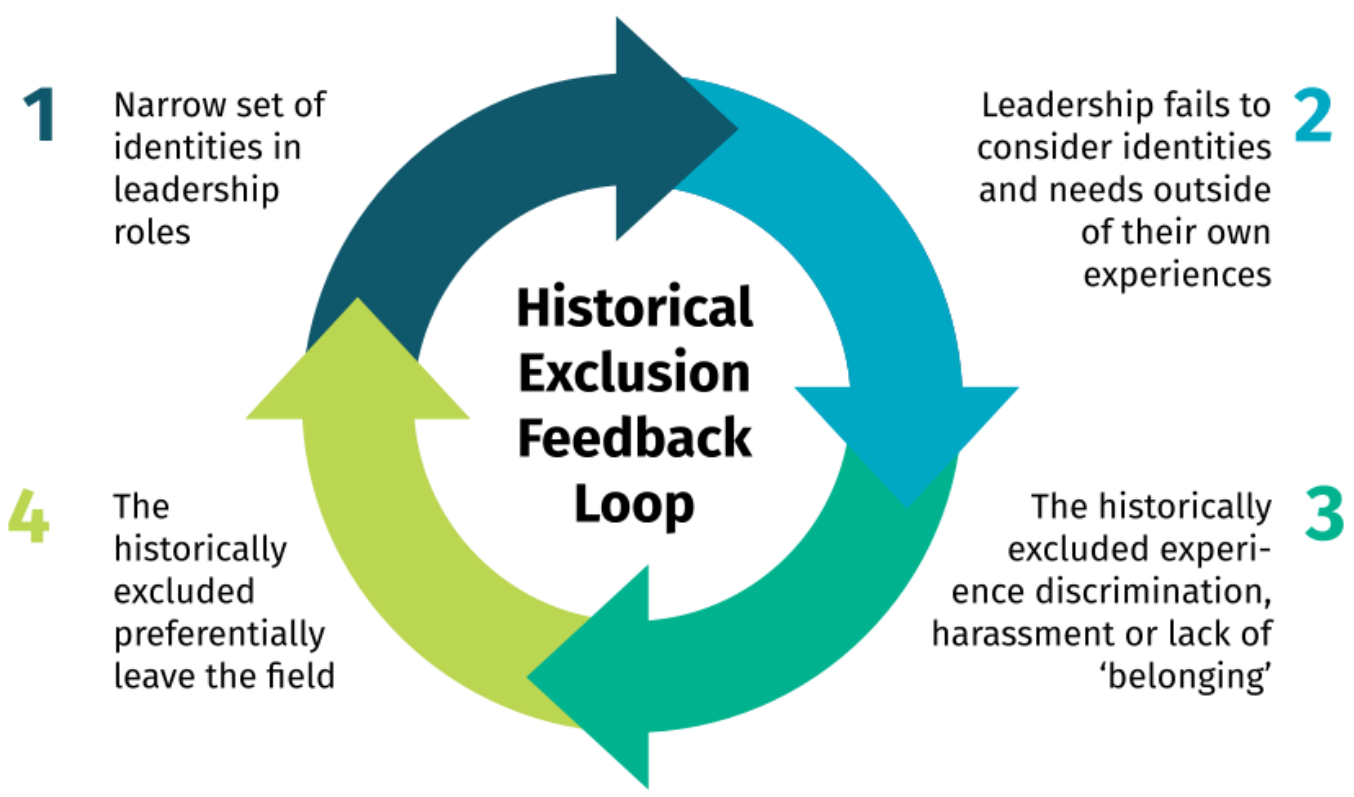

Fig. 1 Historical Exclusion Feedback Loop, illustrating that the ongoing attrition of the historically excluded is not a passive process (Marín-Spiotta et al., 2020) but results from and perpetuates current undiverse, hostile environments in GEES disciplines, which in turn influence careers (Nelson et al., 2017).

\subsection{Belonging}

Beyond just ensuring safety, taught fieldwork should aim to be an experience where all participants feel they belong, and yet "perceptions and subsequent experiences of field teaching are mediated through [...] students' differing backgrounds" (Hughes, 2016, p.461). This is a particular challenge for GEES disciplines in certain parts of the Global North ${ }^{1}$, which are, for example, disproportionately white (Bromery, 1972; Bernard and Cooperdock, 2018; Dowey et al., 2021), and where fieldwork environments can be especially hostile to "individuals who do not conform to the stereotype of the able-bodied, white, cis-gender male rugged field scientist" ${ }^{2}$ (Marín-Spiotta et al.. 2020, p.121). Further, the financial costs associated with fieldwork present income/class barriers to participation (Giles et al.., 2020; Abeyta et al., 2021; Ali et al., 2021). For residential fieldwork, a typical component of GEES education, individual autonomy is reduced compared with normal day-to-day routines. Students, demonstrators, and other staff depend upon the logistics and itinerary planned by someone who may be unfamiliar with their personal needs and lived experiences.

\footnotetext{
${ }^{1}$ This characterisation applies to the UK and to the US, for which GEES diversity data exist, but likely also applies more broadly (e.g. Europe, Canada, Australia/New Zealand).

${ }^{2}$ While this stereotype seems pervasive in many majority white countries in the Global North, different cultures and countries may have differing stereotypes of a (GEES) field scientist.
} 
Participants are "removed from their normal daily structures and their support networks, with possible impacts on wellbeing" (Batty, 2020); further, "exclusion can be even more malign for students of colour as they can neither escape the situation nor seek solace from friends and family at the end of the day who are potent sources of support" (Kraftl et al., 2021, p. 21). Lastly, "inclusion must not require that people advocate for themselves, their own rights, or their own humanity. [...] [Those in the majority] must anticipate the needs of diverse communities and proactively meet them" (Keisling et al.. 2020, p.2, borrowing from the Black queer writer and activist Audre Lorde).

\section{General Tips}

\subsection{Acknowledge Participants' Identities and Backgrounds}

- Identity affects participation, engagement, and learning in the field. For example, racism and isolation in 'off campus' spaces such as field courses were recurring themes in interviews with University of Birmingham GEES undergraduates from minority ethnic backgrounds (Kraftl et al., 2021; Singh et al., in prep.) and "field-based teaching is experienced as a focal site of distress, anxiety and ordeal for many [...] with common mental health conditions" (Tucker and Horton, 2018, p.84). Expect that some of your students have never been in the field before. Expect that some of your students will be international and some will be inexperienced with travel. Expect that some of your students are from cultures or demographics with lower participation in outdoor-based activities and/or from locations that do not have accessible green spaces, while others may have little experience navigating outdoor city spaces. Expect that some of your students grew up in climates that do not experience weather conditions as extreme (cold, hot, snowy, rainy, or dry) as your field course locality. Expect that even minor added or hidden costs represent a financial barrier (Giles et al., 2020; Abeyta et al., 2021; Lawrence and Dowey, 2021). Expect a range of physical fitness levels. Expect that the cohort will include students with gender, sexuality, disability, religious, and/or racial/ethnic minority identities that have been historically excluded from participation in GEES disciplines, and that some of these barriers are invisible (Jackson, 2021). Simple and practical interventions to support access and inclusion will likely benefit all fieldwork participants (e.g., Houghton et al., 2018).

- We each imagine diversity in different and limited ways, so it is important staff take the time to get to know demonstrators and students as best they can before fieldwork commences. Ensure these efforts include international students, students from historically excluded groups, and/or students whose identities differ from your own: "Feeling unable to contact educators can be particularly challenging for minority ethnic students as they feel more isolated intellectually and socially than their White peers [...] so [they] cannot always seek advice from others in their cohort" (Kraftl et al., 2021, p. 14).

- Failure of staff to get to know participants and/or failure to proactively imagine and accommodate diverse and intersecting identities will result in a field experience that, for some participants, creates anxiety, hampers learning outcomes, and may even be 
This paper is a non-peer reviewed preprint submitted to EarthArXiv.

dangerous. Everyone deserves an opportunity to safely participate in fieldwork and good attention to identities in fieldwork planning will facilitate this.

- However, getting to know your students and self-educating on marginalised identities and the corresponding risks should not involve "asking individuals to relive trauma surrounding their identity as a source of education" (Demery and Pipkin, 2020, p.7). Self-education should target literature written by (not about) the identities you seek to understand and support (e.g., Kingsbury et al.. 2020). Equally, recognise that no group is a monolith and individual experiences and needs will differ.

- Lip service is frequently paid to diversity and inclusion in higher education, but the lack of follow-through is manifest. Be aware that historically excluded participants (e.g., Black students) may be operating with a deep pre-existing trust deficit that majoritarian (e.g., white) staff must work hard to bridge.

\subsection{Invite Participants' Questions}

- Specifically and repeatedly invite participants to share individual concerns or questions - including anonymously or in private - and sincerely offer to collaborate with them to adjust fieldwork plans so that they are safe and feel they belong. Many concerns or adjustments may not require, qualify for, or benefit from a laborious formal 'reasonable adjustment' process, but can be actioned via a simple discussion with the student. See section 4.2 .3 for further discussion of the formalised 'reasonable adjustments' process as practiced in UK Higher Education.

- Students asking for accommodations often experience being treated as if they are an inconvenience or a burden. If students approach you about needing accommodations do not create this impression. Reframe your own understanding of the situation before responding: those asking for accommodations are being burdened with laborious, time-consuming (often futile!) self-advocacy for equitable access to learning because systems and practices have not been designed with their identities and needs in mind (see Fig. 1).

- Treat students' concerns seriously and empathetically. Do not dismiss field-related anxieties as disinterest in the subject matter or a lack of willingness to participate. Students may feel afraid to come forward for fear of being perceived as not belonging. This can be exacerbated if staff or students who are more capable and/or experienced with the outdoors associate fieldwork with a toxically masculine, "toughen up" culture. Students' issues should never be dismissed as personal weakness or be met with a "get over it" attitude.

- Some students may wish to share their concerns and needs and others may prefer not to disclose. Anticipating a range of diverse needs and identities beyond those disclosed by students is important for these reasons. The practice of preemptively building in accessibility is the principle of Universal Design for Learning.

\subsection{Distribute Detailed Information Early}

- Providing comprehensive, detailed information up front about all aspects of a field course is a recurrent theme in the recommendations below, e.g. for field guides (section 4.2.13) and pre-fieldwork briefings (section 4.3). Information should include logistic, domestic, and cultural aspects of the travel. There are multiple positive 
This paper is a non-peer reviewed preprint submitted to EarthArXiv.

outcomes for participants and leaders alike when details are given in advance of fieldwork.

- Uncertainties and unknowns are major sources of anxiety in any context, but this is especially so when people visit new places or undertake new activities. Laying out what to expect can go a long way in empowering participants to approach fieldwork with excitement instead of trepidation.

- There are endless kinds of accommodations people need across their lives, and fieldwork participants will only be able to request the relevant ones if they know what is expected of them in the first place. Consider a field site down 100 outdoor stairs and along $500 \mathrm{~m}$ of sandy beach. The entire pathway might be inaccessible to a wheelchair user. Someone with balance problems might feel unsafe about the stairs but able to walk to the field site from a different entry point. Someone with an energy limiting condition might request to walk down the stairs and along the beach but be picked up in a place that avoids the return climb, or they might feel fine doing the entire trek in the morning but not at the end of a day of other activities. Someone with hearing loss or an auditory processing disorder might feel comfortable with the walk but worried about catching instructions at the field site over the noise of waves. Help students help you be inclusive: explain the location, timing, duration, and intensity of activities to participants while there is still ample time for them to request accommodations.

- By providing maximum clarity on fieldwork logistics, students will be able to plan effectively for their own needs, reducing anxiety and the need to divulge sensitive or embarrassing personal information to staff to ensure their own comfort and safety. For instance, when participants know what kit they need and where to get it (section 4.2.13 and section 4.3.4), they can acquire any missing items ahead of time instead of sharing, doing without, or asking instructors for help finding a way to source it during the trip. Detailed information about physical requirements of the fieldwork can also obviate the need for certain accommodations. For example, if the timing, duration, and intensity of a high-exertion activity are clearly explained, a person with insulin-dependent diabetes might independently adjust their dosage and meals an hour or more in advance to stabilise their blood sugar throughout and keep apace. The same person doing the same activity without forewarning might be forced to stop for 20 minutes to treat low blood sugar.

- Writing out fieldwork information the first time will be time-consuming, but this investment can save effort for instructors during the trip and in future iterations of the course. Even if no one comes forward with explicit questions, concerns, or thanks about the fieldwork details, be assured that providing this information still could have ensured the emotional and physical safety of participants.

\section{Checklist}

The following checklist suggests a number of actions to take prior to, during, or after a field course along with details of the rationale underlying the suggested best practices. A condensed, printable version of the checklist action points can be found in section 8 below. 
This paper is a non-peer reviewed preprint submitted to EarthArXiv.

\subsection{Advance Preparation}

\subsubsection{Training}

- Physical first aid training for staff (and sometimes students) is standard, but is also insufficient to ensure the health and safety of all participants. Mental health first aid training (Batty, 2020), bystander intervention training, and antidiscrimination training (Anadu et al., 2020) are necessary to keep historically excluded participants safe. This training should include discussion of microaggressions and microaffirmations (Roberts, 2021). It is strongly recommended that this training be mandatory for all students and staff. Staff and demonstrators must also be trained on university reporting procedures for serious violations (e.g., harassment, abuse).

$\circ$ Request that your institution require these additional types of training if they do not yet do so.

- Ensure that this training is kept up to date.

\subsubsection{Code of Conduct}

- "Field experiences differ according to presence or absence of rules, and consequences if rules were violated" (Nelson et al., 2017, p.713). Develop a standardised, clear Code of Conduct that applies to all participating staff, demonstrators, and students across all fieldwork in your department or university. A zero-tolerance policy for actions such as sexual and racial harassment should be included (Marín-Spiotta, 2018). The Code of Conduct should specify what to do if you experience or witness a violation, including providing guidance about which incidents should be dealt with informally and which must be formally reported. Escalation procedures should be clear and transparent and repercussions for violations should be spelled out. Further, reporting options must include staff members external to the home department/school to avoid conflicts of interest and minimise the likelihood of retaliation. See ADVANCEGeo Partnership: In the Field for example Codes of Conduct.

\subsubsection{Health, Safety, Hazards and Risks}

- Standard hazard and risk assessment forms should be designed to specifically prompt fieldwork leads to consider risks associated with different identities and protected characteristics. See Anadu et al., 2020; Demery and Pipkin, 2020; and Prior-Jones et al., 2020.

- Medical forms should be designed with care. In addition to spelling out privacy and data protection procedures, consider including an explanation of the kinds of medical conditions that are important for field staff to know about and why, and describe how staff will use the information provided to support participants and improve accessibility and inclusion. Keep in mind that international students have very different healthcare systems which may or may not recognise or have very different concepts of certain medical conditions, mental health, and/or disabilities. Providing a clearly-worded explanation may encourage disclosure. These forms should also contain a section for 'what to do if', in case an emergency related to a disability happens. As an example, autistic people can go into sensory overload or people with 
This paper is a non-peer reviewed preprint submitted to EarthArXiv.

anxiety or PTSD may suffer panic attacks very quickly. Individuals will be best placed to know what they need in such situations, which can differ strongly from person to person. Some autistic people go non-verbal when overwhelmed, so it is important to put this kind of information in writing ahead of time.

- Distribute personal medical forms to all students, demonstrators, and staff as far in advance as possible such that information disclosed can be woven into the fieldwork planning.

\subsubsection{Resources}

- To allow the safe and full participation of some students, financial support will be necessary for added or hidden costs and lost wages (see section 4.2.3). Investigate potential funding streams and lobby for financial support at your institution so that these are in place well in advance of taught fieldwork.

- Digital technology can make a major difference to field accessibility and inclusion, but also needs resourcing and testing in advance. See Houghton et al., 2018; Atchison et al., 2019; Whitmeyer et al., 2020.

- Implementing these recommendations on a field course will take time and care. Line managers should allocate sufficient staffing, including reserving ample funding for compensating demonstrators (section 4.2.9) and ensuring workload allocations for permanent staff are truly commensurate with the time required to plan and implement a safe and inclusive field course.

\subsection{Fieldwork Planning}

\subsubsection{Choosing a Destination}

- What are the laws or local attitudes with respect to LGBTQ+ identity? (See e.g., Black. 2019; Murphy. 2020; Jackson, 2021.) What are the laws or local attitudes towards non-majoritarian race, religion, or religious attire (e.g., widespread Islamophobia or countries/regions with laws banning headscarves or face veils)? Laws with respect to alcohol/drinking age? Laws with respect to unmarried couples or sexual intercourse? Is political freedom a concern? See Prior-Jones et al., 2020 for an inclusive risk assessment tool listing further considerations. Some of these factors, particularly geopolitical concerns, can shift rapidly and need to be regularly reevaluated. Further, be aware that for some destinations, particularly in the Global South, more sensitivity and some training will be required so that fieldwork staff, demonstrators, and students respect local culture, e.g., learn some phrases in local languages or how to address locals (particularly elders) respectfully (see Lunn, 2014).

- If your desired destination is international, what kind of visa requirements are there and how do the visa type, application process, and fees vary according to the nationality of the applicant? You may have participants for whom visa procurement will be a lengthy, uncertain, and/or costly process, and which may require additional advance paperwork from the university. You may also have participants with complicated immigration status for whom any international fieldwork will be a barrier and exclusionary. 
This paper is a non-peer reviewed preprint submitted to EarthArXiv.

- To avoid potential exclusion of international students and late arrivals who may need visas, it may be particularly pragmatic to keep fieldwork near the start of the course 'within country'.

- Passports can also present challenges for trans travellers. In some countries it is time-consuming, expensive, or even impossible to change your passport, so the name and particularly the photograph may not resemble the traveller and can cause serious problems at immigration.

- Ideally, visas should be paid for by the organisation rather than the individual to ensure equitable access to fieldwork amongst the cohort.

- In choosing a destination, you may wish to consider whether there are social norms that may negatively impact individual participants. For example, in certain countries minoritised groups, particularly Black people, may be treated as objects of fascination or have their personal space violated; women may be catcalled; and fat people may experience public ridicule. These considerations may or may not rule these destinations out for taught fieldwork, but awareness and empathy around others' experiences is a prerequisite for effective bystander intervention.

- Consider whether your intended destination will present added challenges for those with dietary restrictions (e.g., vegetarians/vegans, participants who keep kosher or halal, or have allergies or intolerances).

\subsubsection{Permission/Ethics}

- Meeting legal requirements is a minimum standard. Check whether any of the sites you plan to visit are protected (this includes biology and environment, not just rocks) and whether your intended activities are permissible. Obtain relevant permissions (e.g., site access). Obtain sampling permission and export/import permits, if applicable.

- Beyond legality, the fieldwork must also be ethical. Remove the "intellectual wall" between the physical landscape and "the humans that inhabit this space" (Pico. 2021, p.12). Whose land is it, are locals involved in your work, and are they mutually benefitting (or at minimum not being harmed) by your visit (North et al., 2020)? Is the land colonised or the ownership of the land disputed? If so, there may be tribal communities whom you should additionally consult and from whom you should seek permission and collaboration (see Gewin, 2021). To ensure your activities are ethical requires considerations beyond just the legal ownership of the land (see e.g., Trisos et al., 2021).

- Undertake an ethical review of your fieldwork plans and complete ethics forms. Discuss any ethical concerns or questions with (e.g.) school or university ethics officers.

- Consider the carbon footprint of the fieldwork, in particular whether the learning objectives can be achieved without flights. More modest carbon footprint reductions can also be achieved through judicious choice of vehicle hire.

\subsubsection{Accessibility}

- Field localities should be accessible to those with a range of physical, sensory, and mental disabilities. Access should not require high levels of physical fitness: "skills 
This paper is a non-peer reviewed preprint submitted to EarthArXiv.

over hills" (Lawrence and Dowey, 2021). Signpost the degree of physical exertion required at each stop in your field guide (see section 4.2.13).

- Facilitate a student's participation as far as possible rather than assigning them an alternative stay-at-home assignment (e.g., an essay). They will miss out on socialisation, camaraderie, team learning, and field experience - this is exclusionary.

- Do not assume that students with disabilities or physical limitations would rather stay home than go in the field. Familiarise yourself with the range of physical abilities of your students to make accommodations for those with limitations (Carabajal et al.. 2017; Atchison et al., 2019) and be aware that a person's abilities may change from day to day.

- Conversely, respect individuals' rights to say no, either to specific activities or whole courses. So long as instructors have done due diligence in providing details about a course (section 3.3), adults should be trusted to make informed assessments of their individual needs and risk profiles and to make decisions accordingly. For instance, people with an identical diagnosis may differ markedly in kinds or degrees of symptoms experienced; only an individual knows their body, limits, and risk aversion level well enough to decide if participation is safe for them. Similarly, people may have different assessments of the risk COVID-19 presents to them, based on their health, personal characteristics, family and living arrangements, or other factors. Peer pressure and instructor-student power dynamics can pressure students to participate who would otherwise opt out. Students who regularly mask or push through mental or physical symptoms may feel particularly reluctant to withhold consent for fieldwork because of fear or past experience of being told their justification is insufficient. Aim to create a class ethos where students feel safe enough they can say no.

- In the UK, students may consult with university disability services to put in place a Reasonable Adjustment Plan (RAP), which outlines the support and reasonable adjustments that are recommended for them over the course of their university studies. As far in advance as possible, obtain and review the RAPs that your students have in place. RAPs should be honoured in the field, but do not presume RAPs alone are sufficient to ensure field participation and accessibility. Many students will not have RAPs in place or even know what adjustments might be useful in the field, especially if they are unfamiliar with fieldwork. The RAP process may also be too inflexible or slow to be useful. Further, international students may be less likely to have RAPs because they are not aware of the RAP system, do not have or cannot provide documentation from their home healthcare system, or are unaware or have different understanding of mental health issues or disabilities. RAPs should be seen as complementary to staff making an effort to get to know their students (see section $\underline{3.1}$ ).

- Presume that each and every added, hidden, or unexpected cost represents a barrier to participation (Giles et al., 2020; Abeyta et al., 2021), including field clothing, personal protective equipment (PPE), technical kit, travel, lodging, food, visa applications, special/extra medication, (international) mobile phone/data charges, and additional care costs. Some participants may also have an additional financial penalty due to lost employment wages while in the field. Make sure students are aware of all 'hidden' costs, give advice about how to source the right gear at a sensible price, and provide advice about what gear is really indispensable. Consider 
This paper is a non-peer reviewed preprint submitted to EarthArXiv.

whether any kit can be purchased by your institution for all students. Advertise (and lobby for) sources of financial support to cover expenses.

- Consider proximity to health support, e.g. health care centres or hospitals, and toilet facilities (Greene et al., 2020).

\subsubsection{Housing Accommodation}

Best practice would be to consult participants about their accommodation needs and preferences well in advance of booking. In practice, this is not always possible as group bookings need to be made far in advance and choice for large cohorts in remote settings may be limited. As far as possible consider the following criteria in making your choice:

- Choose accommodations in settings with good phone/internet access (important for mental health).

- Choose accommodations with some provision of individual rather than shared rooms. Allocating solo rooms to some minoritised individuals may be counterproductive particularly for those experiencing feelings of isolation or social exclusion - as a lot of bonding can take place in shared rooms. However it is likely some participants will need privacy or their own space because of medical reasons, certain disabilities, or neurodiversity (e.g., autism). Sharing accommodation may also be deeply embarrassing or even dangerous for trans or intersex individuals. Do not pry or make individuals feel that they owe you an explanation or a justification as to why they need solo accommodation, as they may not wish to disclose their sex, gender, medical, neurodiversity, or disability status.

- It is important to choose accommodation that is flexible in terms of gender segregation. Participants (especially women) from more conservative households (particularly those who are African, South Asian, and/or Muslim/Sikh/Hindu) may feel more comfortable sharing a room with someone from the same gender out of modesty. There may also be a societal expectation; in some cultures it is frowned upon for a woman to share a room with a man before marriage. However, accommodation that forces participants into a gender binary (e.g. a women's dorm and a men's dorm) is exclusionary for gender nonbinary participants and, further, may present real risks for trans or intersex participants. For this reason, shared rooms/caravans are preferable to dormitories as they allow for a flexible combination of gender-segregated and gender-neutral accommodation.

- Communicate with participants about housing access needs well in advance of booking. By having made an effort to understand participant identities (section 2.1), leaders should be able to anticipate requests like housing for personal assistants, service dog exercise and relief areas, and allowance for a participant to travel a day early so they are rested enough to participate in course activities as fully as possible.

- Full catering may be preferable to self-catered accommodations from a logistics point of view, but, without considered alternatives, can present a barrier for those with specific dietary needs or certain medical conditions. No matter which you choose, it is important to allow students opportunities to purchase and prepare their own food.

- If self-catering:

- Some students may need their own space/utensils (allergies, religious reasons). 
This paper is a non-peer reviewed preprint submitted to EarthArXiv.

- What are the costs? Students may already have university meal plans. Check that they are not paying double for food - this will likely present a significant financial barrier for some.

- Include ample time in the itinerary for grocery shopping, cooking, eating, and cleaning.

- Some students may not be used to catering for themselves and may be embarrassed about this. It is worth checking in to see if anyone needs some advice or support. You might provide links to simple recipes beforehand for students to think about, or suggest kitchen novices share accommodation with more experienced cooks.

- If catered:

- Ensure the full range of dietary requirements can be met as far as possible, and with sufficient variety and nutrition for the activities being undertaken (e.g., vegans not being offered salad every day). This includes meals, but also snacks. Catered accommodations often fall short here, providing prepackaged snacks that many students cannot eat. Be proactive in ensuring the catering meets dietary needs or else take responsibility for filling gaps yourself, e.g., purchasing and distributing vegan or gluten free snacks.

- It is likely some requirements cannot be met (e.g., allergies, intolerances, religious considerations, or aversions) so plan time for supermarket trips.

- If students cannot eat catered food, ensure that they are not paying for it on top of their personal catering costs.

- Group meeting spaces, quiet spaces, and prayer rooms are highly desirable.

- Refrigeration facilities for any medications that need to be kept cold. Mini fridges in rooms can run dangerously cold or inconsistent in temperature, and so participants may feel safer storing medication in a larger refrigerator, e.g., in a hotel kitchen to which leaders need to arrange access.

\subsubsection{Scheduling}

- In addition to factors like seasonal weather and tides, consider religious calendars when scheduling your field dates. Check an interfaith calendar such as https://www.interfaith.org.uk/resources/religious-festivals and ask participants in advance of scheduling whether there are any particular religious festivals that are relevant to them. Consider fasting holidays (e.g., Ramadan or Yom Kippur), holidays where work is not permitted (e.g., Rosh Hashanah) and other major religious holidays (e.g., Christmas, Good Friday, Easter Sunday, Diwali, Eid-ul-Adha, Eid-ul-Fitr, Laylat al-Qadr, Passover/Pesach and Persian New Year (Navroz)) and avoid these dates if possible. The timing of religious holidays may differ between sects or denominations, e.g., the start/end of Ramadan sometimes differs by 1-2 days and orthodox Easter/Christmas fall later in the calendar. If a conflict with a major religious holiday is unavoidable (e.g., Ramadan timing being finalised well after you've made a booking), consider scheduling some down time for participants to celebrate (e.g., attend a local service, if possible, or call friends and family). Further, for fasting holidays, consider making adjustments like slowing the pace of the fieldwork and inviting students to discuss what other adjustments might allow them to 
This paper is a non-peer reviewed preprint submitted to EarthArXiv.

participate safely. If fieldwork spans weekly days of rest/worship (e.g., Fri, Sat, or Sun for Abrahamic religions), consider allowing time to attend a service, if there is a local church, mosque, or synagogue.

- Some religious holidays mandate observances that are impossible in the field, for example, because they require in-person attendance at a religious institution, or fasting may make participation unsafe. If no other arrangements can be made, this should be considered grounds for alternative, desk-based activities. It should be acknowledged that observance varies widely within religions: one person may be comfortable participating in fieldwork with adjustments to allow for religious observance, whereas another of the same religion may be unable to take part in some/all field activities. Therefore, options should be discussed on a case-by-case basis with the participant concerned.

\subsubsection{Health, Safety, Hazards, and Risks}

- Complete a Hazard and Risk assessment

- Consider field safety for all attendees, cognizant of any disabilities or limitations. This will involve reviewing medical forms (see separate bullet point) and considering individuals whose identities pose additional risk. This should be included in your hazard and risk form (see section 4.1.3).

- Review previous years' incident and near miss forms (see section 4.5.2).

- Check what uni travel insurance covers; consider advising students to get their own travel insurance if appropriate.

- Hazard and risk assessment needs to be checked off by a third party and communicated to all participants prior to the activity commencing so that everyone is familiar with the risks and control measures (see section 4.3.3).

- Medical forms

- Field lead (or designated individual with appropriate skills or background) to check completion of student/staff personal medical forms, read them, and alert the staff team about those at risk.

- Ensure hard copies are brought along in the field

- Investigate local emergency services. Whom do you call in a medical emergency? Where is the nearest hospital? Whom would you call if a participant's prescription medication is lost or runs out?

- Next of kin details to be filed as a precaution in case they need to be passed on to emergency room services. (Staff should not discuss students with next of kin or third parties without written consent.)

- Be aware that there will always be 'unknown unknowns'. Students from certain cultures or countries do not have the same awareness or the same definitions as in the UK, particularly around mental health and disabilities. Also bear in mind that disabilities, in particular mental illnesses, are highly stigmatised. Some may be undiagnosed and some may not accept or admit to having a mental illness or disability.

- Obtain copies of your university's emergency action plan/checklist and incident forms from your Health and Safety team. 
This paper is a non-peer reviewed preprint submitted to EarthArXiv.

- Invite students to bring concerns or discuss their needs in private well in advance of a final 'field briefing' (see section 4.3.1). Do not make them feel as if they are an inconvenience or a burden (see section 3.2). Make adjustments to your field course accordingly.

\subsubsection{Code of Conduct Amendments}

- Consider whether any additions to the Code of Conduct (section 4.1.2) specific to your fieldwork destination and itinerary are warranted.

\subsubsection{Combating Isolation}

- Group size may be dictated by student numbers, but a large group can be intimidating and makes building positive, personal working relationships between staff and students and among students more difficult. Consider offering multiple taught fieldwork sessions in parallel or creating small 'field support' groups where a more intimate understanding of individual needs is possible.

- Build in small group exercises. While it is tempting to allow students to simply choose to work with their friends, consider assigning or rotating groups to reduce social isolation, promote cohort bonding, and ensure everyone is included.

- Empower students to help one another (see section 4.3.2 and section 4.2.10).

\subsubsection{Staffing}

- Consider diversity in staffing your field team (including demonstrators) so that students are more likely to have someone they consider 'safe' to whom they can bring concerns. Demonstrating on a field course can also be an important career development opportunity for early career researchers. To ensure equitable access, demonstrators must be fully compensated; partial or insufficient compensation is a barrier to participation. Keep in mind that some individuals may choose not to put themselves forward as demonstrators if they had poor or even traumatic experiences with fieldwork as undergraduates.

- The labour of implementing this checklist should not be outsourced to staff who are themselves from historically excluded groups. This is a form of cultural taxation (e.g., Jimenez et al., 2019; Gewin, 2020).

\subsubsection{Learning Objectives}

- Consider building the lessons from this checklist or the references cited herein into your fieldwork learning objectives and your assessments. You are training the next generation of field scientists and it is critical that they be prepared to apply the lessons in this checklist in their future workplaces. Learning objectives might also include exploring and interrogating the imperialistic legacy of knowledge production and training practices in GEES fieldwork. For example, fieldwork practices today can mimic the marginalising imperialism imposed by 19th century geologists, perpetuating practices of exclusion (Pico, 2021).

- One option would be assigning this checklist and/or some of the cited references as reading and scheduling small group (see section 4.2.8) 
This paper is a non-peer reviewed preprint submitted to EarthArXiv.

discussions and/or assigning short reflective essays as a formatively assessed exercise.

- Consider including formative pre-fieldwork activities in the classroom or on campus to introduce students to what they will be doing in the field. This may help reduce anxiety.

- Include demonstrators in seeing how this checklist is being implemented in your field course. They are an ideal demographic to target for training as future field leaders themselves.

\subsubsection{Logistics, Itinerary, and Kit}

- Allow ample time for walking, learning activities, supermarket trips, meal times, etc. Include ample toilet stops throughout each day (Greene et al., 2020) and ensure that all this information is highlighted in advance (section 4.3.4) and in the field guide (section 4.2.13). Any changes should be communicated and agreed with the students.

- If regular toilet stops are impossible, read the educational primer contained in Greene et al., 2020 and consider carefully whether your preferred itinerary is worth the resulting anxiety, distress, and health risks.

- Plan to stick to reasonable working hours and include ample breaks and down time in the schedule. Overstuffing the schedule is pedagogically ineffective, but also ableist, discriminating against participants with certain disabilities. Some participants may need additional planned or unplanned stops, whether to manage medical conditions, fatigue, or accidents. For instance, people with diabetes, energy limiting conditions, or postural tachycardia syndrome (POTS) may need regular and/or sudden stops to eat a snack or hydrate.

- Transport - coaches, flights, hirecars

- Consider accessibility and inclusion in choosing a mode of transport. If you have flexibility, it is worth bearing in mind that some trans people prefer not to fly in order to avoid dealing with certain airport security procedures, including mismatches between passports and name/appearance (see section 4.2.1), risking non-prescription medicines like hormones being confiscated (self-medicating is common because of the poor state of trans care in the NHS), or risking outing themselves if their prescription hormones are scrutinised at check in (see section 4.3.4 about flying with prescription medications).

- Airline travel can be physically painful for passengers who are fat or have atypical body shapes because airline seats are designed to not fit their bodies. Further, "when fat passengers are seen to be taking up more than their 'fair' share of the space, they become the target of abuse" (Evans et al.. 2021, p.1817). Research the seat width on the air carriers that service your intended destination and strongly consider picking the option with the widest seats. Further, be aware that airlines should carry seat belt extenders for those that need them.

- If you do choose to fly, be aware that some medical conditions, disabilities, phobias, or simply a propensity for travel sickness can make flying physically or emotionally challenging. There may be any number of reasons participants may need costlier exit row seats with extra legroom or aisle seats for easy 
This paper is a non-peer reviewed preprint submitted to EarthArXiv.

access to the bathroom or to be able to get up and stretch. Make sure there are opportunities for participants to make such requests without requiring them to divulge private information.

- Avoid tight connecting flights, which may be more challenging to navigate as a first time flyer, with certain disabilities, or if you have checked baggage.

- Be aware that different airlines have different policies on how they deal with nut allergies and often need to know in advance so they can alter their food offerings and make announcements at the beginning of a flight. There may also be additional requirements such as a doctor's note to bring an epipen on board an aircraft (which may incur an additional cost to obtain from a UK doctor's surgery).

- Are the coaches accessible for participants with physical disabilities? Do the coaches have toilets on board (Greene et al., 2020)? Avoid booking coaches with 3 seats abreast as these seats tend to be narrower.

- Arrange for at least one support vehicle (car) for fieldwork in large groups (e.g., when the main group is travelling by coach) in case of emergencies. Cars can sometimes park closer to the destination than coaches, improving accessibility for participants with certain disabilities.

- Collate kit including maps, GPS, first aid kits, PPE, and additional items like menstruation supplies (see Greene et al., 2020). Ensure inclusive sizing in university-supplied PPE as best you can.

- Consider whether language support is necessary (i.e., translators). The responsibility for translating should not be left to students.

- Consider access to money/cash for students and staff in case of emergency. Perhaps a university credit card? Are there ATMs in the vicinity of the accommodation?

- If participants will be working in small groups, supply each individual with a printed official letter of support (Chaudhary and Berhe, 2020; Demery and Pipkin, 2020), with justifications from a field leader about why they are in a field location, information about hotel and travel bookings, with university branding and contact information, and ideally with a translation in the local language. If this seems unnecessary, keep in mind that people of colour, especially Black people, may not be given the benefit of the doubt by members of the public or by the police (Bowden and Buie, 2021); "Holding objects (e.g., a rock hammer) has been viewed as 'suspicious' and continues to be used as a reason to call the police on Black people" (Ali et al., 2021). It is imperative we protect students most at risk.

\subsubsection{Communication and Delegation}

- Share phone numbers between staff/demonstrators. A group chat (e.g. WhatsApp) may be useful, although it is important to avoid making critical announcements only using channels or platforms that are not universal to all participants. For international fieldwork, have a discussion ahead of time about mobile phone plans, particularly for UK-based field courses heading to the EU post-Brexit, as data and roaming charges will have increased. Consider building some contingency funds into the budget for unexpected emergency mobile charges.

- You may wish to arrange for a non-personal phone/emergency contact number for staff to give students such that staff do not have to distribute their own private phone numbers. This should be supplied by the school or university. 
This paper is a non-peer reviewed preprint submitted to EarthArXiv.

- Designate staff/demonstrator roles.

- Field lead - logistics (keeping to time, coordinating with coach drivers, accommodation personnel, etc.)

- Someone in charge of medical forms

- This person should have up to date data privacy (GDPR) training to ensure documents are held and disposed of properly.

- If the group will be split up at any point, you'll need multiple copies so that one set stays with each group. This is particularly critical in rural or remote settings with poor mobile phone reception.

- First aid lead (in charge of first aid kits)

- Someone in charge of kit

- Someone in charge of documents - handouts, guides, assessment paperwork, student feedback forms

- All staff designated as 'safe person' for students/staff to report concerns

- Follow up process needs to be in place and known to all.

- At least two members of staff/demonstrators to be alcohol free 24 hours a day on a rota basis (or similar). At least one should be a driver. Zero-tolerance alcohol policy for designated drivers.

\subsubsection{Create Inclusive Field Guides}

- Include an explicit timetable for each day, detailing info about toilet facilities, travel times, break times, food/shop availability, and walking duration/difficulty so students can manage expectations/energy and alert staff if they need further accommodations or deviations from the schedule. An example itinerary info sheet is provided in Greene et al., 2020.

- Participants who are preoccupied worrying about the itinerary, logistics, and their own needs will not be active, engaged learners. It is especially important for students with (e.g.) anxiety, ADHD, or autism to know the plan in advance.

- Include a printed copy of the Code of Conduct (section 4.1.2) within your field guide so everyone has access to these policies at all times.

- List emergency contact information (both local and university) including after-hours contacts. Consider including your university's emergency action plan/checklist in the field guide itself.

- Include information on local hazards, typical seasonal weather/climate, and local flora and fauna (particularly if any are possibly dangerous).

- Include information on important local cultural considerations and/or any socio- or geopolitical sensitivities to be aware of.

- Include key phrases in local language(s).

- Include a kit list, specifying what is and what is not provided by the university. Clearly delineate between essential vs. desirable kit, highlight financial aid options and signpost any reduced cost suppliers, university-negotiated discounts, or second hand options (as discussed in section 4.2.3). Further, be aware that inclusive sizing is practically unheard of for most outdoor clothing companies (particularly for women). The famous adage 'There's no such thing as bad weather, only unsuitable clothing' is stigmatising for those that cannot afford or fit into quality outdoor attire.

- Include information about field-friendly options for menstruation and sanitary supplies as part of the kit list (see Greene et al., 2020). 
This paper is a non-peer reviewed preprint submitted to EarthArXiv.

- Include information about prayer room availability.

- If possible, include photos or virtual tours of the accommodation, showing sleeping/bathroom arrangements and cookware available.

- List supermarkets/pharmacies/ATMs near the accommodation, as applicable.

- Ensure that figures and maps print with high contrast and resolution, in colour schemes that will be distinguishable for people with common forms of colour vision deficiency. Online tools like Coblis make it easy to preview the colour accessibility of an image. Images should also be accompanied by text descriptions, either as alt text in digital documents or printed in captions. Not only do image descriptions make graphics accessible to people with vision loss, but also descriptions often help sighted participants quickly understand the relevant takeaways of complex figures! Inspect the alt text in Fig. 1 of this document as an example or read Harvard's digital accessibility tips.

- Field guides should adhere to the same accessibility standards specified for other printed materials in student RAPs (section 4.2.3). For instance, where a student requires lecture notes and exams printed on coloured paper, in large font, or in braille, field course materials should be provided in the same alternative format.

- Include in the field guide a written invitation to discuss concerns with any member of staff, either prior to or during fieldwork.

\subsection{Pre-Fieldwork Student Briefings}

\subsubsection{Offer Advance Small Group or Private Meetings}

- Meetings in small groups or in private can be focussed on concerns of minority ethnic participants and students from other historically excluded demographics. It is likely they have already had real life experiences of discrimination so an open discussion is not out of place. Bringing this up in front of the whole class is delicate because it can focus unwanted attention or scrutiny on individuals, (e.g.) minority ethnic students. On the other hand, it is not appropriate to presume anyone's identity in order to decide whom to invite to such a meeting. A good compromise might be to distribute this checklist to the class, open with a general statement about how identity can affect experiences in the field, and then invite students to meet with the field leader at a later date to discuss. Reassure students that they can speak freely and without repercussions and can request either small group or 1-on-1 discussions.

- Listen to their experiences and concerns and accept them without casting doubts, providing false reassurances, or making assumptions based on your own experiences. The goal is to discuss potential risks and safeguarding measures. If a participant detects limitations in your willingness or ability to appropriately understand their experience, they may offer "abridged, palatable versions of their experiences" (Pico, 2021, p. 9)., such that staff will inherently be limited in knowing how marginalisation modulates participants' experiences and, further, limited in the degree of safeguarding they can provide.

- A poor response is 'I've been to this destination before and never saw anything like that happen so you have nothing to worry about'. Remember, just because you were treated well does not mean that others, particularly 
This paper is a non-peer reviewed preprint submitted to EarthArXiv.

those with marginalised or minoritised identities, will have a similarly positive experience.

- A good response is 'I hear you and I can assure you that I am prepared to step in, should this happen. Let's talk through some possible interventions and mitigation strategies together.' Your bystander training (see section 4.1.1) will help you here.

- Remember not to expect or press anyone to share their past personal trauma.

- Ask whether and how they would like these risks and safeguarding measures broached at the full student pre-fieldwork briefing and respect their wishes.

\subsubsection{Set an Inclusive and Collaborative Tone}

- Encourage the entire field team (staff and demonstrators) to attend and introduce themselves to students at the whole-group briefing.

- Welcome students coming forward with concerns or requests to make sure their individual safety and sense of belonging are taken care of.

- Preemptively address frequently asked questions non-judgmentally. These may include: What if I can't go? What if I'm worried I can't fully participate for whatever reason? Are alternative assessments an option?

- Make clear that they can come to any member of staff, demonstrator, or other student support officer - whomever they feel most comfortable with. Offer anonymous communication channels as well, for instance by distributing a google form where participants can raise questions or concerns that leaders will then clarify to the whole group.

- Make it explicit that asking questions is appropriate and encouraged, even if they concern the course lead; some students may be particularly worried about reporting or questioning an authority, particularly if they come from cultures where this is frowned upon.

- Set/agree expectations about behaviour - including gathering ideas from students and referring to the Code of Conduct). E.g.:

- No smoking/vaping when stood in groups

- Expectations around alcohol consumption

- No drugs apart from those prescribed to an individual or available over the counter

- Set or agree expectations with the students about the aims of the fieldwork.

- Remind students they have a duty to look out for one another - especially when working in independent groups. Bystander training (including discussions of microaggressions and microaffirmations (Roberts, 2021)), and physical and mental health first aid training for students will prepare them for this.

- Encourage students to avoid scrutinising, judging, or focussing unwanted attention on unfamiliar practices, particularly in shared accommodations. Common microaggressions in these settings might include making negative or incessant comments about prayer practices, medical needs, eating practices (including unfamiliar foods), or Black and Mixed Black sleeping practices (e.g., wrapping your head with a scarf or bonnet to protect your hair).

- Prompt an open discussion with students about social isolation on field courses. Acknowledge that fieldwork is polarising - for some it is the best part of their degree, 
This paper is a non-peer reviewed preprint submitted to EarthArXiv.

but others experience it as a source of acute discomfort, particularly with respect to isolation and socialising. Ask students to suggest strategies and ways they might contribute to help make sure no one is left out - either from the work itself or from after hours socialising.

- Where applicable, encourage students to learn basic phrases and sentences in the local language. This is important in emergencies, but also is a sign of respect, even if the country is English speaking or locals understand/speak English.

\subsubsection{Review Health, Safety, Hazards, and Risks}

- Review the Code of Conduct (section 4.1.2) and explicitly discuss reporting channels.

- Review hazard and risk assessment and ethics forms (section 4.2.6).

- Discuss customs/practices of the fieldwork area that students should be aware of, including hazards. Natural hazards and dangerous flora/fauna need to be specifically discussed regardless of whether the fieldwork destination is international or close to the home institution. Keep in mind that some students may be inexperienced with understanding even local hazards (i.e., they may have grown up in a city and spent little time in remote or rural settings or vice versa) and international students may not know whether any local flora/fauna are dangerous.

- Explicitly discuss risks associated with identity (Demery and Pipkin, 2020) with all participants. Remember not to single out individuals or presume their identities. The goal here is to reinforce bystander training (see section 4.1.1).

- Encourage those with relevant disabilities who have smartphones to install apps like 'Medical ID' and 'ICE' that contain information for first responders and 'Emergency chat' that offers communication help in cases where they are not able to communicate verbally (e.g., an overwhelmed autistic person or someone suffering an asthma attack).

- For international field work, discuss important local laws and customs that students should know about and respect.

- When discussing health and safety precautions, consider that the perceptions can be very different between cultures or countries and be careful not to assume knowledge. For example, measures that are ubiquitous in the UK such as PPE may simply not be the expected norm for international students.

\subsubsection{Review Logistics, Itinerary, and Kit}

- Go over the itinerary from the field guide (section 4.2.13) in detail.

- This should include not just fieldwork activities, but also meal times, bathroom breaks, food shopping opportunities etc. (Greene et al., 2020).

- It is important that these discussions break the 'period taboo' and normalise discussions around menstruation in the field, which is an important part of many peoples' field experiences. (See Wroe, 2020 for a UoB undergraduate student perspective.)

- Where you have set times to regiment activities (e.g., tide times, set departures for travel times etc.) ensure the reasons are explained.

- Consider pre-emptively discussing whether any field sites may present a challenge for those with common phobias, including (e.g.) fear of heights 
This paper is a non-peer reviewed preprint submitted to EarthArXiv.

(cliffs) or confined spaces (caves). If you can show images of the field setting in advance this may help allay concerns.

- Include the travel arrangements in your discussions; if travelling internationally, keep in mind that some students may not have been abroad before.

- Advise them about baggage weight limits, what the rules are for bringing food into the destination, and the $100 \mathrm{~mL}$ limits for liquids or gels in carry on luggage.

- Advise any students travelling with medication to keep the medication in its original packaging, bring a copy of any prescriptions, and store both medicines and prescriptions in hand luggage in case checked suitcases are lost. Some medicines are liquids or gels and may exceed the $100 \mathrm{~mL}$ threshold for hand luggage (e.g., hormone gel flasks commonly prescribed to trans people), but prescriptions and/or doctor's notes are typically sufficient evidence for exceptions. The Home Office has further advice in case any medications contain a controlled drug.

- Show external and internal pictures of accommodation, dining room, bedrooms, showers and bathroom facilities, outdoor space and neighbourhood.

- You might remind students that they can bring a supply of their own food/snacks if they are particular about what they eat (subject to customs laws for international fieldwork).

- Do a Google Earth/Street View walk-through - show the surrounding area, where shops are etc., and nearest medical centres

- Go over the kit list, clothing, weather etc.

- Minimise the required kit, as excess kit represents a financial barrier to some.

- Where applicable, educators should disseminate information to students about customs of local dress - particularly for destinations where women's attire is expected to cover more of the body than in the UK. Local collaborators in the destination country may be best placed to provide advice.

- Allocate housing, first accommodating those who have approached staff about needing individual space for whatever reason. There are considerable pros and cons both to assigning rooms and to allowing students to choose their own roommates. The former will reduce the chances of anyone being housed in uncomfortable or hostile situations, however runs a real risk of social isolation for those that do not (or do not yet) have friends in the cohort. If staff assign housing, this allocation must respect that some of the cohort may require gender-segregated rooms. And yet allocation should never be solely on the basis of gender segregation (i.e. "mens' rooms" and "womens' rooms"), especially when the gender of participants is assumed by staff members. Getting assigned allocations right may require a short (GDPR/data privacy-compliant) student questionnaire, asking about their gender (this should be self-declared) and their preferences for gender-segregated or gender-neutral housing (see section 4.2.4). You may also wish to ask students (e.g.) whether they prefer to share with someone who is sociable and likes to go out a lot or someone who would prefer to stay in and read a book. If students choose their own rooms, one option is to ask students to 'buddy up' in twos and threes, with staff allocating these groups, as well as any students who have not identified 'buddies', 
This paper is a non-peer reviewed preprint submitted to EarthArXiv.

into larger rooms. Extreme care must be taken to not place students in situations they are uncomfortable with, through consultation with students. Going forward, more consultation with students is required to resolve best practice, and should form an explicit part of pre-field course discussions.

\subsubsection{Follow Up}

- Invite further questions after the briefing (e.g., post-briefing drop in session).

- Consider sharing this checklist with students. The goals here are:

- It may help overcome trust deficits for historically excluded participants (see section 3.2). If students know you have incorporated the suggestions from this checklist into your fieldwork planning, it may give them confidence that their identity-based safety and belonging concerns have been considered and may also give them confidence in approaching you and your staff team with concerns or for help (Chaudhary and Berhe, 2020).

- The checklist may in and of itself be educational for students about others' identities, safety, and belonging and help those with majoritarian identities to become better allies.

\subsection{In the Field}

\subsubsection{General Conduct}

- As with the pre-fieldwork briefing, reiterate that students are welcome to come forward at any time with concerns about their individual safety or needs. Make clear that they can come to any member of staff or demonstrator - whomever they feel most comfortable with.

- Regularly revisit expectations - including those from students - and refer to Code of Conduct.

- Keep an eye out for harassment, bullying, and inappropriate behaviour - including amongst fellow staff/demonstrators - and report suspected violations as per the Code of Conduct. Address incidents or concerns (e.g., discrimination, harrassment, microaggressions) as they happen. Do not wait until the evening time as the opportunity to correct the situation may have passed. Bystander training will help here (see section 4.1.1).

- Instructors need to keep in mind that some participants (students, but also possibly demonstrators or staff) will have limited experience of fieldwork and residential trips, and will be in unfamiliar surroundings. Have in place plans for obtaining alternative/emergency supplies.

- Allow participants as much control as is possible over their food options. Do not rush mealtime even if the day is behind schedule. Not only do some people eat slowly, but also mealtimes can be essential intervals of rest and recovery for people managing anxieties and fatigue, whether from adjusting to fieldwork for the first time or managing ongoing mental and physical health conditions. Also take care to minimise scrutiny over eating habits, which can be a trigger or source of shame for those with a history of disordered eating. 
This paper is a non-peer reviewed preprint submitted to EarthArXiv.

\subsubsection{Accessible Course Content Delivery}

- As much as possible, avoid communicating important information to big groups in noisy settings or when facing the wind or ocean. Those at the back will struggle to understand, and participants who are D/deaf or hard of hearing or who have an auditory processing disorder will be excluded. Instead, make announcements or explanations at the accommodation, on the coach, or deliver the information one small group at a time in the field.

- If group communication proves challenging at your field site(s), next time consider investing in an audio tour guide system, which has demonstrable benefits for both disabled and nondisabled participants (Houghton et al.. 2018; section 4.1.4). You might also consider basic British Sign Language training for staff and students as part of pre-departure preparation.

- Make sure a member of staff 'brings up the rear' of any long walks or hikes. Make sure the slowest in the group are not overly scrutinised or judged and that they are also given time to rest after exertion (namely, that the group does not immediately set off again once the slowest participants have caught up). Wait for everyone in the group to be ready to engage before giving instructions or announcements. Feeling like one is holding up the group is a frustrating and shameful experience, but it can be turned into an engaging or teaching moment if an instructor at the back of the group is someone naturally conversant and unhurried. For example, rather than monitoring participants at the back until they get up on their feet or rejoin the main group, try sitting down, having a drink of water, and starting a discussion about the landscape features or wildlife observable from there. The point is to have fun and deflect attention from reaching an end destination at a set rate.

- Abide by the timetable outlined in your field guide.

- Clearly communicate (and get consent for) deviations as soon as possible.

- Regularly reiterate the daily itinerary, including next opportunities to purchase food, next toilet stops, what facilities will be like, so students can plan.

- Where you have set times to regiment activities (e.g., tide times, set departures for travel times etc.), ensure participants are given ample and frequent warning.

- Stay alert to the possibility that health conditions which participants manage well in home settings can become unpredictably difficult to control in new environments. For instance, many drugs administered via skin patches or injections metabolise significantly faster when someone is out in the heat, e.g., insulin, nicotine patches, clonidine (used to treat ADHD and high blood pressure), and some hormone replacement therapies (Vanakoski and Seppälä, 1998; Hao et al., 2016). Sudden loss of management control can be frightening, frustrating, or confusing to the person affected, not to mention dangerous. Stay calm and ask how to be supportive, for instance by allowing the participant a short break from activities or an entire day indoors, or by taking them somewhere private with phone reception where they can call their prescribing physician or someone in their support network.

\subsubsection{Socialising and Alcohol}

- Experiences of social isolation were a running theme in interviews with minority ethnic and international GEES undergraduates at UoB (Kraftl et al., 2021; Singh et 
al., in prep.). If you notice that any students seem to be isolated (or perhaps even if you don't), find subtle ways to revisit or remind all students about the inclusion strategies they proposed in the pre-field course open discussion about social isolation (section 4.3.2). In doing so, take care not to focus unwanted attention on individual students.

- Socialising should not revolve around alcohol (Kraftl et al., 2021; Singh et al., in prep.). There are many reasons that people don't drink - never assume why, and don't probe; these conversations are tiring and staff can lead by example to discourage students adopting these behaviours.

- Consider which locations can be used for a dinner or social location so that they are not alcohol-focussed. Pubs are generally associated with drinking, so consider choosing a restaurant or cafe instead. Even better is socialising in a common space at the accommodation, as some participants may be unable to afford to join for paid dinner or drinks. Can you do activities at the accommodation such as board, card, or ball games? If staying at a youth hostel then can you do anything outside?

- Staff and demonstrators should strongly consider modelling desired behaviour for students. Staff choosing to drink during social hours will necessarily limit which students they end up socialising with, as some may not be comfortable socialising in the presence of alcohol. Further, this will likely result in staff preferentially getting to know and developing rapport with students from certain backgrounds, cultures, and demographics - most likely these will be the students most similar to themselves (see section 3.1 about getting to know your students).

- Ensure the presence of non-alcoholic options in all social gatherings and do not limit this to orange and/or apple juice - there are more exciting options that won't make non-drinkers feel like an afterthought.

\subsubsection{Marking}

- While 'recording field observations' is a reasonable learning objective, it is inadvisable to assess field notebooks directly, particularly on the basis of criteria such as neatness or spelling. Some disabilities make it physically difficult to take neat pen and paper notes in field settings, and assessing on this basis may be ableist. As an alternative, consider allowing students to take notes however they wish (including e.g., a tablet or audio recording), and giving them time to organise their notes out of the field. Field notes (recorded in whatever format) should be regarded as a formative necessary skill, where advice, feedback and examples of good practice are a key part of field training. The notes can then be used to feed into a summative assessed piece of work.

- Describing rock colour, or colouring in geologic maps and stratigraphic sections, can be an essential component of certain field exercises. Remind students that the purpose of noting colour is for a field researcher's own, internal reference. There is no 'right answer' when it comes to describing colours or other sensory experiences, and notebooks should be marked accordingly.

\subsubsection{Contingency Expenses}

- Emergencies (e.g. if a participant needs to leave the field course prematurely due to a medical issue or family emergency) may incur extra costs, which some participants 
This paper is a non-peer reviewed preprint submitted to EarthArXiv.

may be unable to afford up front even if they are eligible for insurance reimbursement at a later date. Field course staff should ensure that participants do not need to pay out of pocket for such expenses, i.e., by arranging for the university/university travel agent to make a travel booking or paying with a university credit or debit card.

\subsection{On Return}

\subsubsection{Respect Participants' Privacy}

- Hard copies of confidential medical information should be shredded/disposed of as confidential waste.

\subsubsection{Reflect and Revise}

- Complete incident forms, including near misses. There are legal obligations about the timeframe for filing these, so check with your Health and Safety team.

- Provide opportunities for students to feed back on the course content as well as personal safety, wellbeing, and belonging. Offer anonymous feedback channels like a google form.

- Schedule an extensive debrief with all staff and demonstrators with particular attention paid to safety and belonging. Reflect on timing, order of activities, preparation, learning outcomes, communication, etc.

- Use student feedback and the staff debrief to make a list of planned changes to improve the field course for the following year.

- Revise the field guide, hazard and risk assessment, and pre-briefing materials/presentation while the experience is fresh in your mind.

- Keep good notes - this is particularly important in case the field course is run by a different person in the future.

\subsubsection{Iterate and Self-Educate}

- Getting this stuff right is a constant work in progress. Reflect on student feedback, on situations that you and your team did not feel prepared to handle or did not feel you handled well, and use these as a starting point for directing further self-education and training prior to the next field course.

\section{Acknowledgements}

Thanks to Farzana Azad (University of Birmingham alumna), Rebecca Bartlett (University of Birmingham), Lesley Batty (University of Birmingham), Ellen Cliff (University of Oxford), Isabel Fenton (University of Oxford), Wai Tung Ng (University of Birmingham alumna), and Nussaïbah Raja (Friedrich-Alexander-Universität Erlangen-Nürnberg) for a number of helpful comments and suggestions.

\section{Further Resources}

\section{Unlearning Racism in Geoscience resource list}


This paper is a non-peer reviewed preprint submitted to EarthArXiv.

International Association for Geoscience Diversity resource list

ADVANCEGeo Partnership resource list

Royal Geographical Society accessible fieldwork resource list

Royal Geographical Society research conduct and field ethics resource list

Considering International Mobility for LGBT+ Scientists (Royal Society of Chemistry)

Global mobility scheme, University of Birmingham Rainbow Network

Global Code of Conduct for Research in Resource-Poor Settings

UK government Lesbian, Gay, Bisexual and Transgender foreign travel advice

\section{References}

Abeyta, A., Fernandes, A.M., Mahon, R.C., and Swanson, T.E., 2021, The True Cost of Field Education is a Barrier to Diversifying Geosciences:, doi:10.31223/X5BG70.

Ali, H.N. et al., 2021, An actionable anti-racism plan for geoscience organizations: Nature Communications, v. 12, p. 1-6, doi:10.1038/s41467-021-23936-w.

Anadu, J., Ali, H., and Jackson, C., 2020, Ten steps to protect BIPOC scholars in the field: Eos, v. 101, doi:10.1029/2020EO150525.

Atchison, C.L., Marshall, A.M., and Collins, T.D., 2019, A multiple case study of inclusive learning communities enabling active participation in geoscience field courses for students with physical disabilities: Journal of Geoscience Education, v. 67, p. 472-486, doi: $10.1080 / 10899995.2019 .1600962$.

Black, R., 2019. Queer voices in palaeontology. Nature; doi: $\underline{10.1038 / d 41586-019-02113-6}$.

Batty, L., 2020, Mental health and fieldwork: Geological Society of London blog, https://blog.geolsoc.org.uk/2020/05/19/mental-health-and-fieldwork (accessed July 2021).

Bernard, R.E., and Cooperdock, E.H., 2018, No progress on diversity in 40 years: Nature Geoscience, v. 11, p. 292-295, doi:10.1038/s41561-018-0116-6.

Bowden, A.K., Buie, C.R., 2021, Anti-Black racism in academia and what you can do about it, Nature Reviews Materials, doi:10.1038/s41578-021-00361-5.

Bromery, R., 1972, Minorities in the geosciences: American Geological Institute: Geotimes, v. $17(2)$, p. $23-24$.

Carabajal, I.G., Marshall, A.M., and Atchison, C.L., 2017, A synthesis of instructional strategies in geoscience education literature that address barriers to inclusion for students with disabilities: Journal of Geoscience Education, v. 65, p. 531-541, doi:10.5408/16-211.1.

Chaudhary, V.B., Berhe, A.A., 2020, Ten simple rules for building an antiracist lab: PLoS Computational Biology, v. 16(10), p. e1008210, doi:10.1371/journal.pcbi.1008210.

Clancy, K.B., Nelson, R.G., Rutherford, J.N., and Hinde, K., 2014, Survey of academic field experiences (SAFE): Trainees report harassment and assault: PloS one, v. 9, p. e102172, doi:10.1371/journal.pone.0102172. 
This paper is a non-peer reviewed preprint submitted to EarthArXiv.

Cooperdock, E.H., Chen, C.Y., Guevara, V.E., and Metcalf, J.R., 2021, Counteracting systemic bias in the lab, field, and classroom: AGU Advances, v. 2, p. e2020AV000353, doi:10.1029/2020AV000353.

Demery, A.-J.C., and Pipkin, M.A., 2021, Safe fieldwork strategies for at-risk individuals, their supervisors and institutions: Nature Ecology \& Evolution, v. 5, p. 5-9, doi: $\underline{10.1038 / s 41559-020-01328-5}$.

Dowey, N. et al., 2021, A UK perspective on tackling the geoscience racial diversity crisis in the Global North: Nature Geoscience, v. 14, p. 256-259, doi:10.1038/s41561-021-00737-w.

Elkins, J.T., and Elkins, N.M., 2007, Teaching geology in the field: Significant geoscience concept gains in entirely field-based introductory geology courses: Journal of geoscience education, v. 55, p. 126-132, doi:10.5408/1089-9995-55.2.126.

Evans, B., Bias, S., and Colls, R., 2021, The Dys-Appearing Fat Body: Bodily Intensities and Fatphobic Sociomaterialities When Flying While Fat: Annals of the American Association of Geographers, p. 1-24, doi:10.1080/24694452.2020.1866485.

Gewin, V., 2020, The time tax put on scientists of colour: Nature, v. 583, p. 479-481, doi:10.1038/d41586-020-01920-6.

Gewin, V., 2021, How to include Indigenous researchers and their knowledge: Nature, v. 589, p. 315-317, doi:10.1038/d41586-021-00022-1.

Giles, S., Jackson, C., and Stephen, N., 2020, Barriers to fieldwork in undergraduate geoscience degrees: Nature Reviews Earth \& Environment, v. 1, p. 77-78, doi: $10.1038 / s 43017-020-0022-5$.

Gilley, B., Atchison, C., Feig, A., and Stokes, A., 2015, Impact of inclusive field trips: Nature Geoscience, v. 8, p. 579-580, doi:10.1038/ngeo2500.

Greene, S., Ashley, K., Dunne, E., Edgar, K., Giles, S., and Hanson, E., 2020, Toilet stops in the field: An educational primer and recommended best practices for field-based teaching: OSF Preprints, doi:10.31219/osf.io/gnhj2.

Hao, J., Ghosh, P., Li, S. K., Newman, B., Kasting, G. B., and Raney, S. G., 2016, Heat effects on drug delivery across human skin: Expert Opinion on Drug Delivery, v. 13, p. 755-768, doi:10.1517/17425247.2016.1136286.

Houghton, J.J., Morgan, D.J., Gordon, C.E., Stokes, A., Atchison, C.L., Collins, T.D., Craven, B., and Willis, K., 2020, Access Anglesey 2018: Lessons from an inclusive field course: Advances in Geosciences, v. 53, p. 183-194, doi:10.5194/adgeo-53-183-2020.

Hughes, A., 2016, Exploring normative whiteness: ensuring inclusive pedagogic practice in undergraduate fieldwork teaching and learning: Journal of Geography in Higher Education, v. 40, p. 460-477, doi:10.1080/03098265.2016.1155206.

Jackson, C., 2021, Some barriers are invisible: Geoscientist Magazine, p. 16, https://geoscientist.online/sections/viewpoint/some-barriers-are-invisible 
This paper is a non-peer reviewed preprint submitted to EarthArXiv.

Jimenez, M.F., Laverty, T.M., Bombaci, S.P., Wilkins, K., Bennett, D.E., and Pejchar, L., 2019, Underrepresented faculty play a disproportionate role in advancing diversity and inclusion: Nature ecology \& evolution, v. 3, p. 1030-1033, doi:10.1038/s41559-019-0911-5.

John, C.M., and Khan, S.B., 2018, Mental health in the field: Nature Geoscience, v. 11, p. 618-620, doi:10.1038/s41561-018-0219-0.

Kingsbury, C.G., Sibert, E.C., Killingback, Z., and Atchison, C.L., 2020, "Nothing about us without us:" The perspectives of autistic geoscientists on inclusive instructional practices in geoscience education: Journal of Geoscience Education, v. 68, p. 302-310, doi: $10.1080 / 10899995.2020 .1768017$.

Kraftl, P., Singh, S., and Pykett, J., Guisse, A., Hodgson, E., Humelnicu, U. E., Keen, N., Kéïta, S., McNaney, N., Menzel A., N'Dri, K., N'Goran, K.J., Oldknow, G., Tiene, R. and Weightman, W., 2021, Understanding the experiences of students from minority ethnic backgrounds to explain the degree awarding gap: University of Birmingham School of Geography, Earth and Environmental Sciences internal research report.

Lawrence, A., and Dowey, N.J., 2020, Six simple steps towards making GEES fieldwork more accessible and inclusive, doi:10.31223/X5VG81.

Lunn J. ed., 2014, Fieldwork in the Global South: Ethical challenges and dilemmas. Routledge, doi:10.4324/9780203096895.

Marín-Spiotta, E., Barnes, R.T., Berhe, A.A., Hastings, M.G., Mattheis, A., Schneider, B., and Williams, B.M., 2020, Hostile climates are barriers to diversifying the geosciences: Advances in Geosciences, v. 53, p. 117-127, doi:10.5194/adgeo-53-117-2020.

Marín-Spiotta, E., 2018, Harassment should count as scientific misconduct: Nature, v. 557(141), doi:10.1038/d41586-018-05076-2.

Mogk, D.W., and Goodwin, C., 2012, Learning in the field: Synthesis of research on thinking and learning in the geosciences, in Kastens, K.A. and Manduca, C.A. eds., Earth and Mind II: A Synthesis of Research on Thinking and Learning in the Geosciences, Geological Society of America, v. 486, p. 0, doi:10.1130/2012.2486(24).

Murphy, V., 2020, Geoscience course stops running Oman fieldtrip to be more inclusive: Imperial College London,

https://www.imperial.ac.uk/news/198915/geoscience-course-stops-running-oman-fieldtrip (accessed July 2021).

Nelson, R.G., Rutherford, J.N., Hinde, K. and Clancy, K.B.H., 2017, Signaling Safety: Characterizing Fieldwork Experiences and Their Implications for Career Trajectories: American Anthropologist, v. 119, p. 710-722, doi:10.1111/aman.12929.

North, M.A., Hastie, W.W., and Hoyer, L., 2020, Out of Africa: The underrepresentation of African authors in high-impact geoscience literature: Earth-Science Reviews, v. 208, p. 103262, doi:10.1016/j.earscirev.2020.103262.

Olcott, A., and Downen, M., 2020, The challenges of fieldwork for LGBTQ+ geoscientists: Eos, v. 101, doi:10.1029/2020EO148200. 
This paper is a non-peer reviewed preprint submitted to EarthArXiv.

Petcovic, H.L., Stokes, A., and Caulkins, J.L., 2014, Geoscientists' perceptions of the value of undergraduate field education: GSA Today, v. 24, p. 4-10, doi:10.1130/GSATG196A.1.

Pico, T., 2021, Linking Past to Present in a Postcolonial Field Science: How Scientific Training and Practice in US Geology Perpetuates Marginalization, doi:10.1002/essoar.10506337.1.

Pickrell, J., 2020, Scientists push against barriers to diversity in the field sciences: Science, v. 374 , p. 375 , doi:10.1126/science.caredit.abb6887.

Prior-Jones, M., Pinnion, J., Millet, M.-A., Bagshaw, E., Fagereng, A., and Ballinger, R., 2020, An inclusive risk assessment tool for travel and fieldwork, EGU General Assembly 2020, Online, 4-8 May 2020, EGU2020-7678, doi:10.5194/egusphere-egu2020-7678.

Roberts, 2021, Use microaffirmations and call out microaggressions to help others, Nature Career Column, doi:10.1038/d41586-021-01498-7.

Scarlett, J.P., 2021, A minority within a minority. Nature Reviews Earth \& Environment, v. 2, p. 455 , doi: $10.1038 / \mathrm{s} 43017-021-00184-6$.

Singh, S., Pykett, J., Kraftl, P., Guisse, A., Hodgson, E., Humelnicu, U. E., Keen, N., Kéïta, S., McNaney, N., Menzel A., N'Dri, K., N'Goran, K.J., Oldknow, G., Tiene, R. and Weightman, W., 2021, [in prep.] Understanding the 'degree awarding gap' in geography, planning, geology and environmental science in UK higher education through peer research.

Trisos, C.H., Auerbach, J., and Katti, M., 2021, Decoloniality and anti-oppressive practices for a more ethical ecology: Nature Ecology \& Evolution, p. 1-8, doi:10.1038/s41559-021-01460-w.

Tucker, F., and Horton, J., 2019, "The show must go on!" Fieldwork, mental health and wellbeing in Geography, Earth and Environmental Sciences: Area, v. 51, p. 84-93, doi:10.1111/area.12437.

Vanakoski, J., and Seppälä, T., 1998, Heat exposure and drugs: Clinical Pharmacokinetics, v. 34, p. 311-322, doi:10.2165/00003088-199834040-00004.

Viglione, G., 2020, Racism and harassment are common in field research-scientists are speaking up. Nature, v. 585(7823), p.15-16, doi:10.1038/d41586-020-02328-y.

Whitmeyer, S.J., Atchison, C., and Collins, T.D., 2020, Using mobile technologies to enhance accessibility and inclusion in field-based learning: GSA Today, v. 30, doi:10.1130/GSATG462A.1.

Wroe, M., 2020, Invisible barriers to fieldwork: breaking the period taboo, SATNAV Magazine (Science and Technology News and Views), https://www.satnavmag.com/post/invisible-barriers-to-fieldwork-breaking-the-period-taboo (accessed August 2021). 


\section{Printable Checklist: Safety and Belonging in the Field}

\section{Advance Preparation (section 4.1)}

Arrange physical first aid training, mental health first aid training, and bystander intervention training for all staff and students

Write/update a Code of Conduct, including clear escalation procedures and repercussions

Write/update risk assessments, including risks from personal characteristics

Write/update personal medical forms to encourage disclosure

Distribute personal medical forms, risk assessments, liability waivers, travel insurance, and any other necessary paperwork to all participants, including staff and demonstrators

Learn about, advertise, or lobby for sources of financial support for course attendance

\section{Fieldwork Planning (section 4.2)}

Check current laws/attitudes of intended destination towards various protected groups

Liaise with participants about visa requirements or other travel documents if needed

Secure ethical approval to work at field sites with legal protections or tribal sovereignty

Read any Reasonable Adjustment Plans; work with participants to plan accommodations

Choose housing with as much flexibility as possible, e.g. including single and shared rooms, prayer space access, and proximity to medical care, supermarkets, and shops

Arrange meals-assemble cooking teams or pass on dietary requirements to caterers

Consult a multi-faith calendar, tide tables, local holiday dates, etc. to schedule fieldwork

Print hard copies of medical forms and emergency protocols for staff

Print official letters of support for participants to have on hand when separated from staff

Assemble a staff team with diverse experience, identities, and expertise

Create lesson plans; consider group-work, pre-fieldwork preparation exercises, and building lessons from this checklist into assessments.

Build an itinerary around working hours, with breaks, toilet stops, and food/shopping trips 
This paper is a non-peer reviewed preprint submitted to EarthArXiv.

Book transport with a range of bodies in mind and with ample time for connections

Collect kit, e.g., high-vis jackets, first aid kits, and loanable field clothing

Share contact information within the staff team and designate leads for first aid, kit, etc.

Add domestic and emergency information to field guides: Code of Conduct, extensive itinerary, risk assessments, contacts, key phrases in local language(s), facilities list, etc.

\section{Pre-Fieldwork Student Briefings (section 4.3)}

Reserve bookable office hours in anticipation of private or small group meetings to address questions, concerns, or accommodations

Invite all instructors/demonstrators to attend the main briefing and introduce themselves

Mutually agree expectations and aims for fieldwork and participant culture (e.g., involving learning objectives, alcohol, and acceptance towards different personal practices). Include an open discussion about strategies for students to help one another combat social isolation.

Review risk assessments, Code of Conduct, and local laws or customs with participants

Walk through the itinerary including transport and housing arrangements, e.g., using Google Earth or Street View and pictures of facilities

List the minimum required kit and options for acquiring it

Allocate rooms, negotiating varied needs for privacy, same-sex rooms, and buddy groups

Share this checklist document with participants to educate and build trust

\section{In the Field (section 4.4)}

Handle any inappropriate behaviour according to Code of Conduct

Review the itinerary with participants, including activities, facilities, and breaks

Preserve scheduled breaks, including full lunchtimes-if this means falling behind schedule, make a note for future years to reduce activity time

Wait for everyone to arrive and prepare at a site before announcing information

Assign a staff member to bring up the rear of the group; leave no one behind

Opt for alcohol-free settings for meals and social events

Ensure at least 2 staff members stay alcohol-free at all times 
This paper is a non-peer reviewed preprint submitted to EarthArXiv.

Opt not to mark field notebooks directly or, at minimum, mark them without regard to handwriting, neatness, or spelling accuracy; allow notes in alternative formats

\section{On Return (section 4.5)}

Shred confidential participant information

Complete incident report forms

Send anonymous feedback form to participants

Schedule instructor/demonstrator debrief session

$\square$ Revise field guide, risk assessment, and pre-briefing materials/presentation for next use 